\title{
Human Telesupervision of a Fleet of Autonomous Robots for Safe and Efficient Space Exploration
}

\author{
Gregg Podnar \\ Carnegie Mellon University \\ 5000 Forbes Ave. \\ Pittsburgh PA 15213-3890 USA \\ $+1-412-268-6273$ \\ gwp@cmu.edu
}

\author{
John Dolan \\ Carnegie Mellon University \\ 5000 Forbes Ave. \\ Pittsburgh PA 15213-3890 USA \\ $+1-412-268-7988$ \\ jmd@cs.cmu.edu
}

\author{
Alberto Elfes \\ Jet Propulsion Laboratory \\ 4800 Oak Grove Drive \\ Pasadena CA 91109-8099 USA \\ $+1-818-393-9074$ \\ elfes@jpl.nasa.gov
}

\author{
Marcel Bergerman, H. Benjamin Brown, Alan D. Guisewite \\ Carnegie Mellon University, 5000 Forbes Ave., Pittsburgh PA 15213-3890 USA
}

\begin{abstract}
In January 2004, NASA began a bold enterprise to return to the Moon, and with the technologies and expertise gained, press on to Mars. The underlying Vision for Space Exploration calls for a sustained and affordable human and robotic program to explore the solar system and beyond; to conduct human expeditions to Mars after successfully demonstrating sustained human exploration missions on the Moon. The approach is to "send human and robotic explorers as partners, leveraging the capabilities of each where most useful.” Human-robot interfacing technologies for this approach are required at readiness levels above any available today. In this paper, we describe the HRI aspects of a robot supervision architecture we are developing under NASA's auspices, based on the authors' extensive experience with field deployment of ground, underwater, lighterthan-air, and inspection autonomous and semi-autonomous robotic vehicles and systems.

This work was supported by NASA under CA \#NNA05CP96A.
\end{abstract}

\section{Categories and Subject Descriptors}

I.4.0 [Image Processing and Computer Vision]: General image displays.

H.5.2 [Information Interfaces and Presentation]: User Interfaces - graphical user interfaces, screen design.

J.2 [Computer Applications]: Physical Sciences and Engineering - aerospace.

\section{General Terms}

Measurement, Performance, Design, Reliability, Experimentation.

\section{Keywords}

Robot supervision architecture, Autonomous navigation, Telesupervision, Telepresence, Teleoperation, Field deployment, Technology readiness.

\section{INTRODUCTION}

Robots have been proven to work very well in extraterrestrial environments, being able to travel autonomously, explore,

Copyright is held by the author/owner(s).

HRI'06, March 2-3, 2006, Salt Lake City, Utah, USA.

ACM 1-59593-294-1/06/0003. photograph, collect samples, and even perform limited scientific analysis of materials. It is therefore desirable that robotic systems, with their proven resilience to the harsh space environment and autonomous capabilities, be used to augment the most precious resource in space, the human beings and their reasoning abilities.

The authors are exploiting this scenario of human-supervised robotic exploration and work by creating and developing the technologies necessary for the deployment of a fleet of robots that operate as autonomously as possible, and have the human supervisor provide direct control only when necessary. This work will result in a general and widely applicable architecture for human supervision of a fleet of autonomous robots in support of sustained, affordable, and safe space exploration. In this paper, we focus on the human-robot fleet interaction aspects of our architecture, which were designed primarily based on the authors' experience with field deployment of ground, underwater, lighterthan-air, and inspection autonomous and semi-autonomous robotic vehicles and systems.

At its highest level of abstraction the Robot Supervision Architecture (RSA) supports human supervision of a fleet of autonomous robots. The human interacts with the system in three ways: making high-level plans and assignments to the robots; monitoring the progress as the robots carry out their assigned plans autonomously; and intervening when a robot requires assistance, whether navigational, or with respect to its assigned task. These are respectively handled by the three top-level processes shown in Figure 1 - Task Planning and Monitoring, Robot Telemonitoring, and Telepresence and Teleoperation.

\section{RSA HUMAN INTERACTION MODES}

The physical embodiment of the RSA's human-robot interaction is the Telesupervisor Workstation, located in a human habitat base, depicted in Figure 2. At its center is the live stereoscopic video display, which faithfully reproduces the scene as if the operator were viewing through a window on one of the robot vehicles. The workstation also provides two other groupings of displays: those which support task planning and monitoring (Figure 2, left); and those which allow continuous robot telemonitoring through a "dashboard" providing low-bandwidth imagery and telemetry (Figure 2, right). 


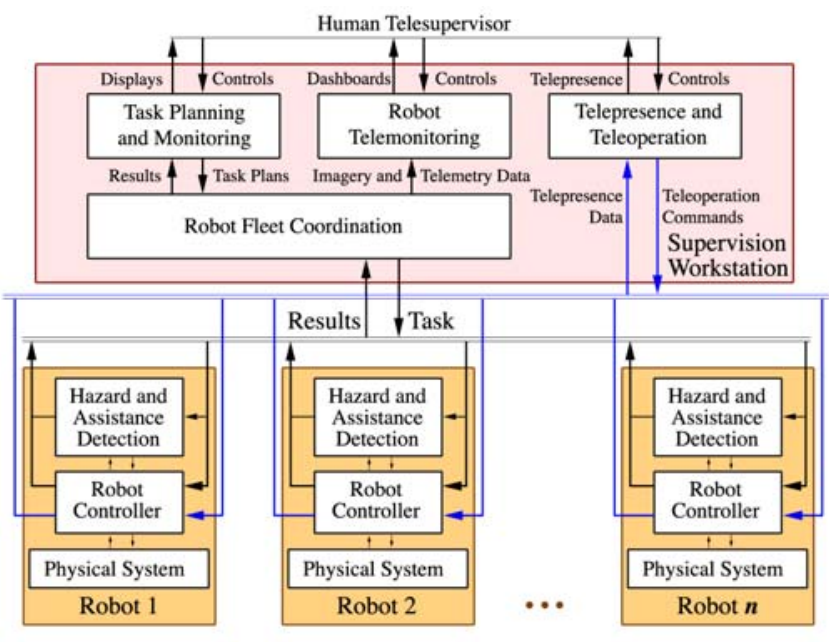

Figure 1. RSA system-level block diagram.

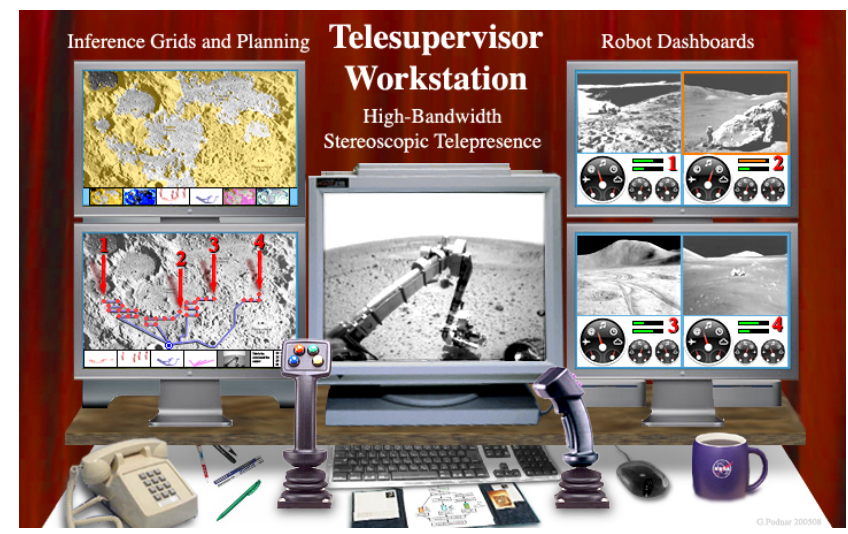

Figure 2. Workstation for human interface with the RSA.

\section{TELEPRESENCE AND \\ TELEOPERATION SYSTEM}

The major challenge of a telepresence system is to provide an endto-end vision system which reduces operator fatigue while enhancing the operator's ability to interact with the remote environment. The system must therefore faithfully reproduce the scene as if it were viewed by the operator's naked eyes. This is not as trivial as mounting two cameras in a toed-in configuration. The human interpupillary distance and eye-screen distance must be considered to determine the camera sensor geometries and lens fields of view. Founded on our previous work and on our understanding of the human binocular visual system, we have designed a geometrically-correct binocular stereoscopic vision system with a stereoscopic camera for each fleet robot, and a stereoscopic display for the operator's workstation. The prototype stereoscopic camera has been successfully integrated with the rover and the prototype telesupervisor workstation, which currently allows for local teleoperation of the CMU K10 rover as well as for remote teleoperation of NASA JPL and NASA ARC rovers. Figure 3 presents the camera mounted on a mast on the K10 along with its CAD drawing and initial prototype. Figure 4 shows the initial instantiation of the workstation with the operator wearing the passive circularly polarized eyewear and driving the rover through the stereoscopic view provided by a StereoGraphics Z-Screen and View/Record unit.
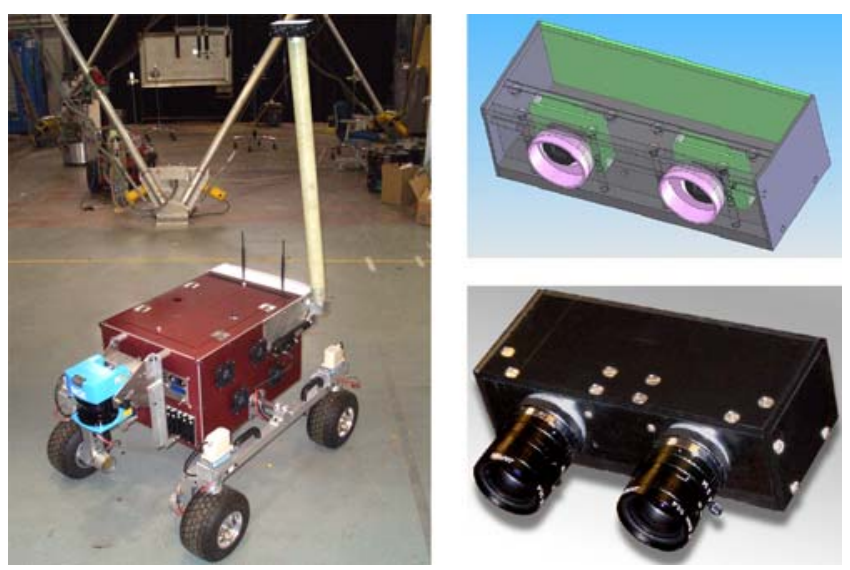

Figure 3. CMU's K10 and stereoscopic camera.

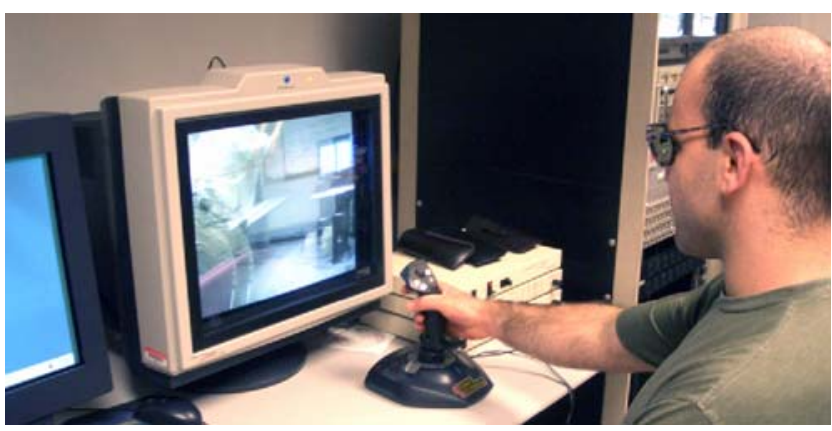

Figure 4. Initial workstation with stereoscopic telepresence capability.

Telemetry from each robot is provided to the telesupervisor via a graphical "Dashboard". CMU's K10 robot includes an inertial measurement unit, a GPS, and a battery charge gauge. The low bandwidth reporting of vehicle health and attitude data supports the telesupervisor to maintain an operational context for each robot in the fleet (Figure 5).

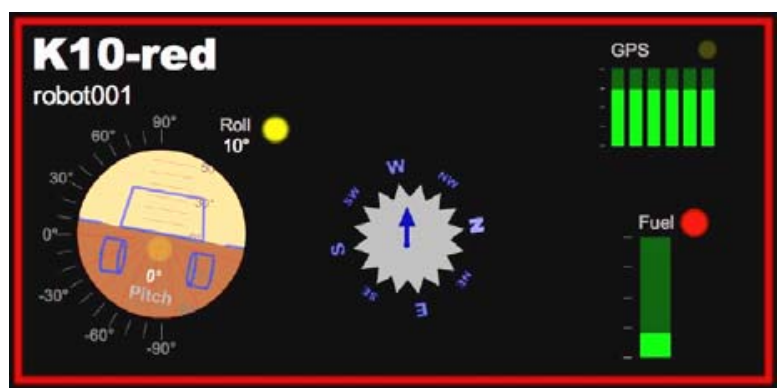

Figure 5. Prototype "Dashboard” for one robot.

\section{CONCLUSION}

The paradigm of a human-supervised fleet of autonomous robots is directly applicable to a variety of tasks in planetary prospecting, mining and construction, as well as to space assembly, inspection, and maintenance. The RSA will be continuously advanced so as to be demonstrated for many of these important applications. Further information on this project, including other publications, can be found at http://www.ri.cmu.edu/ prospect. 